Pacific Journal of Mathematics

ERGODICITY IN YON NEWMAN ALGEBRAS 


\title{
ERGODICITY IN VON NEUMANN ALGEBRAS
}

\section{Charles RAdiN}

\begin{abstract}
We investigate the ergodicity of elements of a von Neumann algebra $\mathfrak{A}$ under the action of an arbitrary cyclic group of inner *-automorphisms of $\mathfrak{A}$. A simple corollary of our results is the following characterization: $A$ von Neumann algebra $\mathfrak{A}$ is finite if and only if for each $A \in \mathfrak{A}$ and inner *automorphism $\alpha$ of $\mathfrak{A}$, there exists $\bar{A} \in \mathfrak{A}$ such that $1 / N \sum_{n=0}^{N-1} \alpha^{n}(A) \underset{N \rightarrow \infty}{\longrightarrow} \bar{A}$ in the weak operator topology.
\end{abstract}

1. Introduction. Our purpose is to explore in a new direction the ergodic theory of von Neumann algebras presented by Kovács and Szücs [2]. In [2] the essential contribution was the introduction of a certain restriction (called $G$-finiteness) on a group of *-automorphisms of a von Neumann algebra, fashioned so that all elements of the algebra behave ergodicly with respect to the group. Instead we consider the action of a natural class of (cyclic) groups of *automorphisms, namely the inner ones, and investigate which elements of the algebra behave ergodicly with respect to all such groups.

2. Behavior of infinite projections. From the ergodic theory developed in [2], we note the following simple consequence.

Theorem 0. (Kovács and Szücs). Let $\mathfrak{U}$ be a finite von Neumann algebra. For each $A \in \mathfrak{X}$ and each inner *automorphism $\alpha$ of $\mathfrak{X}$, there exists $\bar{A} \in \mathfrak{Z}$ such that $1 / N \sum_{n=0}^{N-1} \alpha^{n}(A) \underset{N \rightarrow \infty}{\longrightarrow} \bar{A}$ in the strong operator topology.

Our first result is a complement to this and provides a new characterization of finiteness for von Neumann algebras.

THEOREM 1. Let $\mathfrak{2}$ be a von Neumann algebra. For each nonzero infinite projection $P \in \mathfrak{A}$ there exists an infinite projection $\theta \in \mathfrak{A}$, $\theta \leqq P$, and a unitary $U \in \mathfrak{A}$, such that $1 / N \sum_{n=0}^{N=1} U^{n} \theta U^{-n}$ does not converge in the weak operator topology.

First we need the following lemma.

LEMma. There exists a nonzero properly infinite projection $P^{\prime} \leqq P$.

Proof. Let $S$ be the set of all central projections $E$ of $\mathfrak{A}$ such 
that $E P$ is finite. $0 \in S$ so $S$ is not empty. Let $\left\{E_{\alpha}\right\}$ be an orthogonal family of elements of $S$. If $\sum_{\alpha} E_{\alpha} P \sim Q \leqq \sum_{\alpha} E_{\alpha} P$ (where $\sim$ is the usual equivalence relation for projections in $\mathfrak{Q})$, then $E_{\alpha} P \sim E_{\alpha} Q \leqq E_{\alpha} P$ so that $E_{\alpha} Q=E_{\alpha} P$ and therefore $Q \geqq \sum_{\alpha} E_{\alpha} Q=\sum_{\alpha} E_{\alpha} P$. Therefore, $Q=$ $\sum_{\alpha} E_{\alpha} P$ and $\sum_{\alpha} E_{\alpha} P$ is finite. It follows easily that there exists a (unique) maximal element $F$ in $S$. From [1, III.2.3.5] it follows that $(I-F) P$ is nonzero and infinite. Assume it is not properly infinite. Then from [1, III.2.5.9] there exists a central projection $G$ such that $0 \neq G(I-F) P$ is finite. But then from [1, III.2.3.5] $F<F+$ $G(I-F) \in S$, which contradiction proves our lemma with $P^{\prime} \equiv(I-F) P$.

Proof of Theorem 1. From [1, III.8.6.2] there exists a set $\left\{P_{n} \mid n \in Z\right\}$ of nonzero projections $P_{n} \in \mathfrak{M}$ such that $P_{n} P_{m}=\delta_{n, m} P_{n}$ and $P_{n} \sim P_{m}$ for all $m, n \in Z$, and such that $\sum_{|n| \leqq m} P_{n} \underset{m \rightarrow \infty}{\longrightarrow} P^{\prime}$ in the strong operator topology. Therefore, there exist $V_{n} \in \mathfrak{A}$ such that $V_{n}{ }^{*} V_{n}=P_{n}$ and $V_{n} V_{n}{ }^{*}=P_{n+1}$ for all $n \in Z$, so that $P_{n+1} V_{n}=V_{n} P_{n}$ and $P_{n} V_{n}{ }^{*}=V_{n}{ }^{*} P_{n+1}$ for all $n \in Z$. Define for each $f \in \mathscr{X}$ (the Hilbert space of definition of $\mathfrak{A}$ ),

$$
U f=\left(\operatorname{norm} \lim _{m \rightarrow \infty} \sum_{|n| \leqq m} V_{n} P_{n} f\right)+\left(I-P^{\prime}\right) f,
$$

where the limit exists since $\left\|V_{n} P_{n} f\right\|=\left\|P_{n} f\right\|$ and $V_{n} P_{n} f=P_{n+1} V_{n} f$ so that $\left\{V_{n} P_{n} f \mid n \in Z\right\}$ are pairwise orthogonal and

$$
\sum_{|n| \leqq m}\left\|V_{n} P_{n} f\right\|^{2}=\sum_{\mid n ! \leqq m}\left\|P_{n} f\right\|^{2} \leqq\left\|P^{\prime} f\right\|^{2} .
$$

In fact $U$ is clearly a linear and norm preserving surjection, and therefore unitary. Now since

$$
\left(\sum_{k \mid \leqq l} V_{k} P_{k}\right) \text { norm } \lim _{m \rightarrow \infty} \sum_{|n| \leqq m} P_{n} f=\sum_{\mid n j \leqq l} V_{n} P_{n} f
$$

it follows that $U_{l} \equiv I-P^{\prime}+\sum_{\mid k_{1} \leq l} V_{k} P_{l}$ has $U$ as a strong operator limit as $l \rightarrow \infty$. Therefore, $U \in \mathfrak{A}$. It also follows that $U P_{n} U^{-1}=P_{n+1}$ for all $n \in Z$, and so by induction $U^{m} P_{n} U^{-m}=P_{n+m}$ for all $m, n \in Z$. Now define $g: N \rightarrow\{0,1\}$ by

$$
g(n)= \begin{cases}1 & \text { if } 3^{2 m} \leqq n<3^{2 m+1} \quad \text { for some } m \in N \\ 0 & \text { if } 3^{2 m+1} \leqq n<3^{2 m+2} \text { for some } m \in N .\end{cases}
$$

Then define $\theta$ as the strong operator limit as

$$
K \rightarrow-\infty \quad \text { of } \quad \sum_{m=K}^{0} g(-m) P_{m},
$$

and let $\psi$ be a unit vector in $P_{0} \mathscr{H}$. Now consider 


$$
\begin{aligned}
\left\langle\psi, 1 / N \sum_{n=0}^{N-1} U^{n} \theta U^{-n} \psi\right\rangle & =1 / N \sum_{n=0}^{N-1}\left\langle\psi, U^{n} \theta U^{-n} P_{0} \psi\right\rangle \\
& =1 / N \sum_{n=0}^{N-1} \sum_{m=-\infty}^{0} g(-m)\left\langle\psi, P_{n+m} P_{0} \psi\right\rangle \\
& =1 / N \sum_{n=0}^{N-1} g(n) .
\end{aligned}
$$

It is easy to see that for all $M \in N, 1 / 3^{2 M+1} \sum_{n=0}^{3^{2 M+1}-1} g(n) \geqq 2 / 3$ yet $1 / 3^{2 M+2} \sum_{n=0}^{3^{2 m+2}-1} g(n) \leqq 1 / 3$, and the theorem is proven.

Using Theorem 0 , we have immediately,

CoRollary 1 (resp.2). A von Neumann algebra $\mathfrak{A}$ is finite if and only if for each $A \in \mathfrak{A}$ and inner *automorphism $\alpha$ of $\mathfrak{A}$, there exists $\bar{A} \in \mathfrak{A}$ such that $1 / N \sum_{n=0}^{N-1} \alpha^{n}(A) \underset{N \rightarrow \infty}{\longrightarrow} \bar{A}$ in the weak (resp. strong) operator topology.

3. Finite elements. Theorem 1 raises the question of the ergodic behavior, under arbitrary inner *-automorphisms, of "finite elements" of infinite von Neumann algebras. The following theorem gives some information in this direction.

THEOREM 2. Let $\mathfrak{A}$ be a von Neumann algebra and $\tau$ a faithful normal semi-finite trace on $\mathfrak{X}^{+}$invariant under the *automorphism $\alpha$ of $\mathfrak{A}$. Then for each $A \in \mathfrak{U}$ such that $\tau\left(A^{*} A\right)<\infty$, there exists $\bar{A} \in \mathfrak{A}$ such that $1 / N \sum_{n=0}^{N-1} \alpha^{n}(A) \underset{N \rightarrow \infty}{\longrightarrow} \bar{A}$ in the strong operator topology.

Proof. First we define the following (standard) objects: see e.g. $[1$, I.6.2.2]

$$
\begin{aligned}
& \|\|_{2}: A \in \mathfrak{X} \longrightarrow\left[\tau\left(A^{*} A\right)\right]^{1 / 2} \\
& \mathscr{N}=\left\{A \in \mathfrak{X}\|\| A \|_{2}<\infty\right\} .
\end{aligned}
$$

Let $L_{2}$ be the abstract completion of $\mathscr{N}$ in the norm \|\|$_{2}$, and extend \|\|$_{2}$ to $L_{2}$ in the usual way. Let $i$ be the isometric embedding of $\mathscr{N}$ into $L_{2} \cdot L_{2}$ is a Hilbert space with the obvious addition and scalar multiplication, and inner product $<$, $>$ defined as the extension to $L_{2} \times L_{2}$ of

$$
\tau: A \times B \in \mathscr{N} \times \mathscr{N} \longrightarrow \tau\left(A^{*} B\right) .
$$

We note the simple inequalities

$$
\begin{array}{ll}
\|A B\|_{2} \leqq\|A\|\|B\|_{2} & \text { for all } B \in \mathscr{N}, A \in \mathfrak{U} \\
\|A B\|_{2} \leqq\|A\|_{2}\|B\| & \text { for all } B \in \mathscr{N}, B \in \mathfrak{U} .
\end{array}
$$


We then define the $C^{*}$-representation $\pi$ of $\mathfrak{A}$ on $L_{2}$ by

$$
\pi(A) i(B) \equiv i(A B)
$$

and noting that $\|\pi(A) i(B)\|_{2}=\|A B\|_{2} \leqq\|A\|\|B\|_{2}$ so that $\pi(A)$ extends uniquely to $L_{2}$ by continuity. It is easy to see that $\pi$ is faithful and normal and that

$$
U: i(B) \longrightarrow i(\alpha[B]) \quad \text { for } B \in \mathscr{N}
$$

extends to a unitary operator on $L_{2}$. Defining, for $B \in \mathfrak{A}$,

$$
B_{N}=\frac{1}{N} \sum_{n=0}^{N-1} \alpha^{n}(B) \text {, we know by von Neumann's }
$$

mean ergodic theorem that for each $A \in \mathscr{N}, i\left(A_{N}\right)$ is \|\|$_{2}$-Cauchy. Define for each $B \in \mathscr{N}$,

$$
D_{A}: i(B) \longrightarrow \text { norm } \lim _{N \rightarrow \infty} \pi\left(A_{N}\right) i(B)
$$

which limit exists since

$$
\left\|\pi\left(A_{N}-A_{M}\right) i(B)\right\|_{2} \leqq\left\|A_{N}-A_{M}\right\|_{2}\|B\| \cdot
$$

$D_{A}$ is obviously linear. Furthermore,

$$
\left\|D_{A} i(B)\right\|_{2}=\lim _{N \rightarrow \infty}\left\|\pi\left(A_{N}\right) i(B)\right\|_{2} \leqq\|A\|\|B\|_{2}
$$

so $D_{A}$ extends uniquely to a bounded operator on $L_{2}$ by continuity. It is easy to see that $\pi\left(A_{N}\right)$ converges to $D_{A}$ in the strong operator topology. Since $\pi$ is normal, $\pi(\mathfrak{Q})$ is strong operator closed [1, I.4.3.2] so there exists $\bar{A} \in \mathfrak{X}$ such that $D_{A}=\pi(\bar{A})$. Since $\pi$ is faithful, $A_{N} \underset{N \rightarrow \infty}{\longrightarrow} \bar{A}$ in the strong operator topology [1, 1.4.3.1].

CoRollary 1. Let $\mathfrak{A}$ be a countably decomposable von Neumann algebra. For each finite projection $P \in \mathfrak{X}$ and inner *-automorphism $\alpha$ of $\mathfrak{A}$, there exists $\bar{P} \in \mathfrak{X}$ such that

$$
\frac{1}{N} \sum_{M=0}^{N-1} \alpha^{n}(P) \underset{N \rightarrow \infty}{\longrightarrow} \bar{P} \text { in the strong operator topology . }
$$

Proof. Let

$$
A \in \mathfrak{X} \longrightarrow A_{1} \oplus A_{2} \in \mathfrak{N}_{1} \oplus \mathfrak{N}_{2}
$$

be the canonical decomposition of $\mathfrak{X}$ into its countably decomposable semi-finite and purely infinite components. From [1, I.6.7.9] we know that any finite countably decomposable von Neumann algebra has a faithful, normal, tracial state. Inserting this fact into the proof of 
[3, 2.5.3], we see that there exists a countable faithful family $\left\{\tau_{n} \mid n \in N\right\}$ of normal semi-finite traces on $\mathfrak{U}_{1}^{+}$with pairwise orthogonal supports such that $\tau_{n}\left(P_{1}\right)<\infty$ for all $n \in N$. Define

$$
\tau^{\prime}=\sum_{n=0}^{\infty} \tau_{n} /\left[\tau_{n}\left(P_{1}\right)+2\right]^{n}
$$

on $\mathfrak{U}_{1}^{+}$; it is faithful, normal and semi-finite. Since $\alpha$ is also inner for $\mathfrak{U}_{1}$ and therefore leaves $\tau^{\prime}$ invariant, we may apply Theorem 2 to $\mathfrak{N}_{1}$. Since $P_{2}=0$ from [1, III.2.4.8], we are finished.

In the countably decomposable case, Theorem 2 gives us an essentially different proof of Theorem 0 , namely

CoRollary 2. Let $\mathfrak{A}$ be a finite countably decomposable von Neumann algebra. For each $A \in \mathfrak{U}$ and inner *-automorphism $\alpha$ of $\mathfrak{A}$, there exists $\bar{A} \in \mathfrak{X}$ such that

$$
\frac{1}{N} \sum_{n=0}^{N-1} \alpha^{n}(A) \underset{N \rightarrow \infty}{\longrightarrow} \bar{A} \text { in the strong operator topology . }
$$

Proof. Just combine the existence of a faithful finite normal trace on $\mathfrak{X}^{+}[1$, I.6.7.9] with Theorem 2.

\section{REFERENCES}

1. J. Dixmier, Les algèbres d'opérateurs dans l'espace Hilbertien, 2nd Ed., Paris, 1969.

2. I. Kovács and J. Szücs, Ergodic type theorems in von Neumann algebras, Acta Sci. Math., 27 (1966), 233-246.

3. S. Sakai, $C^{*}$-Algebras and $W^{*}$-Algebras, New York, 1971.

Received June 6, 1972. Research supported by AFOSR under Contract F44620-71-C-0108. PRINCETON UNIVERSITY 



\section{PACIFIC JOURNAL OF MATHEMATICS}

\section{EDITORS}

RICHARD ARENS (Managing Editor)

University of California

Los Angeles, California 90024

R. A. Beaumont

University of Washington

Seattle, Washington 98105
J. DUGUNDJI*

Department of Mathematics

University of Southern California

Los Angeles, California 90007

D. Gilbarg and J. Milgram

Stanford University

Stanford, California 94305

\section{ASSOCIATE EDITORS}
E. F. BECKENBACH
B. H. NeumanN
F. WOLF
K. YosHIDA

\section{SUPPORTING INSTITUTIONS}

UNIVERSITY OF BRITISH COLUMBIA
CALIFORNIA INSTITUTE OF TECHNOLOGY
UNIVERSITY OF CALIFORNIA
MONTANA STATE UNIVERSITY
UNIVERSITY OF NEVADA
NEW MEXICO STATE UNIVERSITY
OREGON STATE UNIVERSITY
UNIVERSITY OF OREGON
OSAKA UNIVERSITY

UNIVERSITY OF BRITISH COLUMBIA

UNIVERSITY OF CALIFORNIA

MONTANA STATE UNIVERSITY

UNIVERSITY OF NEVADA

OREGON STATE UNIVERSITY

OSAKA UNIVERSITY
UNIVERSITY OF SOUTHERN CALIFORNIA

STANFORD UNIVERSITY

UNIVERSITY OF TOKYO

UNIVERSITY OF UTAH

WASHINGTON STATE UNIVERSITY

UNIVERSITY OF WASHINGTON

\section{AMERICAN MATHEMATICAL SOCIETY} NAVAL WEAPONS CENTER

* C. R. DePrima California Institute of Technology, Pasadena, CA 91109, will replace J. Dugundji until August 1974. 


\section{Pacific Journal of Mathematics}

\section{Vol. 48, No. $1 \quad$ March, 1973}

Jan Aarts and David John Lutzer, Pseudo-completeness and the product of Baire

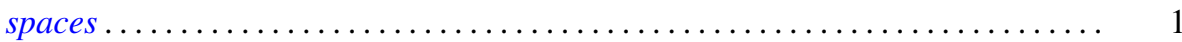

Gordon Owen Berg, Metric characterizations of Euclidean spaces ............ 11

Ajit Kaur Chilana, The space of bounded sequences with the mixed topology ..... . 29

Philip Throop Church and James Timourian, Differentiable open maps of

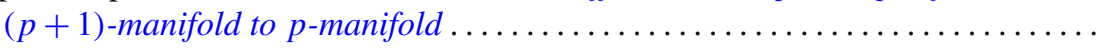

P. D. T. A. Elliott, On additive functions whose limiting distributions possess a finite

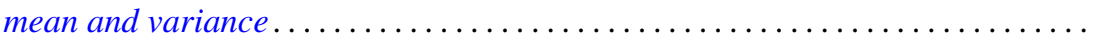

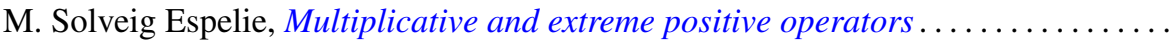

Jacques A. Ferland, Domains of negativity and application to generalized convexity

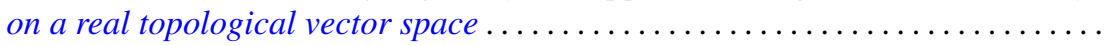

Michael Benton Freeman and Reese Harvey, A compact set that is locally holomorphically convex but not holomorphically convex ...............

Roe William Goodman, Positive-definite distributions and intertwining

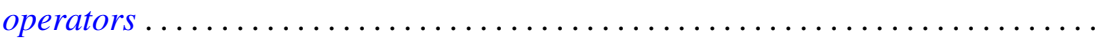

Elliot Charles Gootman, The type of some $C^{*}$ and $W^{*}$-algebras associated with

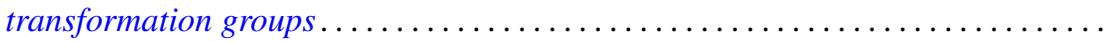

David Charles Haddad, Angular limits of locally finitely valent holomorphic

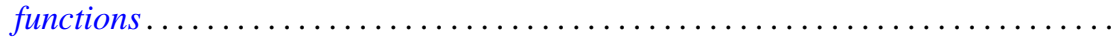

William Buhmann Johnson, On quasi-complements .

William M. Kantor, On 2-transitive collineation groups of finite projective spaces...

Joachim Lambek and Gerhard O. Michler, Completions and classical localizations of right Noetherian rings

Kenneth Lamar Lange, Borel sets of probability measures ......

David Lowell Lovelady, Product integrals for an ordinary differential equation in a Banach space

Jorge Martinez, A hom-functor for lattice-ordered groups .........

W. K. Mason, Weakly almost periodic homeomorphisms of the two sphere ....

Anthony G. Mucci, Limits for martingale-like sequences .......

Eugene Michael Norris, Relationally induced semigroups ...

Arthur E. Olson, A comparison of c-density and $k$-density ......

Donald Steven Passman, On the semisimplicity of group rings of linear groups.

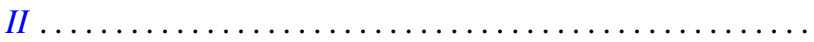

Charles Radin, Ergodicity in von Neumann algebras .

P. Rosenthal, On the singularities of the function generated by the Bergman operator of the second kind.

Arthur Argyle Sagle and J. R. Schumi, Multiplications on homogeneous spaces,

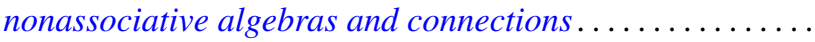

Leo Sario and Cecilia Wang, Existence of Dirichlet finite biharmonic functions on

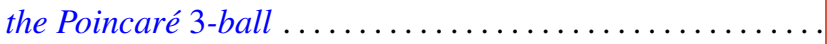

Ramachandran Subramanian, On a generalization of martingales due to Blake ..

Bui An Ton, On strongly nonlinear elliptic variational inequalities.

Seth Warner, A topological characterization of complete, discretely valued

fields. 